\title{
Imaging Information
}

National Cancer Institute

\section{Source}

National Cancer Institute. Imaging Information. NCI Thesaurus. Code C81256.

A collection of data pertinent to a radiographic study. 